\title{
LOOKING INTO CALIPSO CLIMATOLOGICAL PRODUCTS: EVALUATION AND SUGGESTIONS FROM EARLINET
}

\author{
Nikolaos Papagiannopoulos ${ }^{1 *}$, Lucia Mona ${ }^{1}$, Lucas Alados-Alboledas ${ }^{2}$, Vassilis Amiridis ${ }^{3}$, Daniele \\ Bortoli $^{4}$, Giuseppe D'Amico ${ }^{1}$, Maria Joao Costa ${ }^{4}$, Sergio Pereira ${ }^{4}$, Nicola Spinelli ${ }^{5}$, Ulla Wandinger ${ }^{6}$, \\ and Gelsomina Pappalardo ${ }^{1}$ \\ ${ }^{1}$ Consiglio Nazionale delle Ricerche - Istituto di Metodologie per l'Analisi Ambientale (CNR- \\ IMAA),Potenza, Italy,*Email: nikolaos.papagiannopoulos@imaa.cnr.it \\ ${ }^{2}$ Andalusian Institute for Earth System Research, University of Granada (IISTA-CEAMA), Spain \\ ${ }^{3}$ Institute for Astronomy, Astrophysics, Space Applications and Remote Sensing, National Observatory of \\ Athens, Greece \\ ${ }^{4}$ Centro de Geofísica de Évora (CGE), Évora, Portugal \\ ${ }^{5}$ Consorzio Nazionale Interuniversitario per le Scienze Fisiche della Materia, Napoli Research Unit, Italy \\ ${ }^{6}$ Leibniz Institute for Tropospheric Research, Leipzig, Germany
}

\begin{abstract}
CALIPSO (Cloud-Aerosol Lidar and Pathfinder Satellite Observations) Level 3 (CL3) data were compared against EARLINET (European Aerosol Research Lidar Network) monthly averages obtained by profiles during satellite overpasses. Data from EARLINET stations of Évora, Granada, Leipzig, Naples and Potenza, equipped with advanced multi-wavelength Raman lidars were used for this study. Owing to spatial and temporal differences, we reproduced the CL3 filtering rubric onto the CALIPSO Level 2 data. The CALIPSO monthly mean profiles following this approach are called CALIPSO Level $3^{*}$, CL3*. This offers the possibility to achieve direct comparable datasets. In respect to CL3 data, the agreement typically improved, in particular above the areas directly affected by the anthropogenic activities within the planetary boundary layer. However in most of the cases a subtle CALIPSO underestimation was observed with an average bias of $0.03 \mathrm{~km}^{-1}$. We investigated the backscatter coefficient applying the same screening criteria, where the mean relative difference in respect to the extinction comparison improved from 15.2\% to $11.4 \%$. Lastly, the typing capabilities of CALIPSO were assessed outlining the importance of the correct aerosol type (and associated lidar ratio value) assessment to the CALIPSO aerosol properties retrieval.
\end{abstract}

\section{INTRODUCTION}

The CL3 product aims at characterizing the global 3-D distribution of aerosol as well as aerosol seasonal and interannual variations, and ultimately, assisting aerosol models. The main outputs are profiles of aerosol extinction coefficient at $532 \mathrm{~nm}$ and its vertical integral (aerosol optical depth; AOD). CL3 data are mapped onto a globally uniform $2{ }^{\circ} \times 5^{\circ}$ (latitude $\mathrm{x}$ longitude) grid cell. The vertical resolution of the product is $60 \mathrm{~m}$ over the range of the heights -0.5 $\mathrm{km}$ to $12 \mathrm{~km}$ relative to the mean sea level. Monthly mean extinction profiles are computed for four conditions: nighttime/daytime all-sky and cloud-free. In addition, several quality control flags contained in the Level 2 files are used to screen the data prior to averaging. A detailed summary of the methodology used for the production of the CL3 product is provided in the Appendix of [1].

It is important to quantitatively evaluate the accuracy of any data product by comparison with independent measurements. CALIOP is firstly and foremost a profiling instrument; therefore there is nothing equivalent than comparison with profiling data. In any case, a detailed evaluation of CALIPSO extinction profiles (rather than AODs) using ground-based Raman lidars would be the ideal way to evaluate the satellite product. In the frame of the European Aerosol Research Network (EARLINET), various studies dedicated to evaluate CALIPSO Level 1 and Level 2 data have been carried out (e.g., [2]; [3]). So far, few studies 
have been published regarding the CL3 dataset, such as reported from Winker et al. (2013), a comparison with High Spectral Resolution Lidar (HSRL) data given by airborne measurements during CALIPSO underflights of the NASA B200 aircraft (e.g., [4]). Moreover, a thorough assessment of CL3 product is given in [5], where the authors used multi-platform and model data to investigate its performance. Here for the first time, the performance of CL3 data is systematically compared with extinction profiles on continental scale through EARLINET correlative measurements.

\section{METHODOLOGY}

The CL3 data were compared against EARLINET profiles, however the correlative measurements regime and the CALIPSO mapping scheme combined with variable and unfavorable atmospheric conditions resulted in a very dissimilar number of collected observations, namely 272 CALIPSO overflights and 96 EARLINET correlative measurements. Hence, the dataset under examination posed doubts about the statistical significance and the representativeness of the aerosol monthly situation. Consequently, the standard CL3 dataset was compromised to correspond to the simultaneous EARLINET observations. Like this, we achieve to create direct comparable datasets, using only CALIPSO data segments corresponding to collected EARLINET measurements.

The CALIPSO Level 2 Version 3.01 Aerosol Profile product, which includes aerosol extinction and backscatter coefficient profiles at $532 \mathrm{~nm}$ is used to produce the monthly CALIPSO profiles. To ensure that only quality assured Level 2 data are included, the data were screened and low confidence retrievals were excluded, following the CL3 rubric as long as possible. More specifically each $5-\mathrm{km}$ profile was processed according to screening criteria reported in the study of [6]. Differences with CL3 quality screening on the existing steps are related to the Extinction_Coefficient_Uncertainty_532 filter, which is set to a more conservative value. A further step was applied following the A6 of the Appendix of [1]. Regarding the CALIPSO monthly averaging process, a value of $0.0 \mathrm{~km}^{-1}$ is assigned in each profile to layers where the above criteria are invoked or no retrieval was made above $2.5 \mathrm{~km}$. Moreover, the portion of extinction below the range bin that meets those conditions is excluded.

In this analysis quality screened CALIPSO extinction profiles at $532 \mathrm{~nm}$ are directly compared to corresponding EARLINET correlative measurements. Only the nighttime CALIPSO segment is used given the EARLINET ability of nighttime extinction measurements. For the purpose of this work the equivalent monthly mean profile is created whence at least two measurements are available within a period of a month. As far as the sky condition of the measurements, EARLINET cloud free and cirrus cloud infected data are integrated in the statistics, whilst for CALIPSO cloud free and above cloud data were employed. The reason for this disparity comes from the nature of the systems.

Typically EARLINET performs correlative measurements while the satellite ground-track lies in a $100 \mathrm{~km}$ radius area from the measuring site. The CL3* grid cell, therefore, corresponds to a grid which is different from CL3 one, and is dictated by the correlative measurements schedule as shown in Figure 1. The latitude boundaries of the new grid are kept the same as CL3.

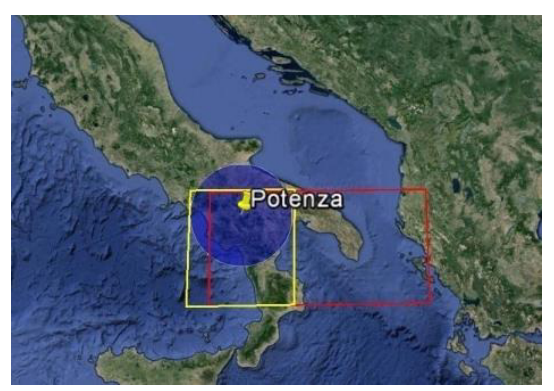

Figure 1: Spatial boundaries of the CALIPSO dataset for Potenza cell. Red frame shows the CL3 grid containing Potenza EARLINET station (yellow pin), the corresponding CL3* grid is designated by the yellow frame. The blue circle has a radius of $100 \mathrm{~km}$ and represents the area that triggers measurements upon the satellite overflight.

\section{RESULTS}

This section reports as example results of the proposed methodology to the Potenza cell. The Potenza EARLINET site is a mountainous site and is located in the middle of the Mediterranean, its relatively low local aerosol content makes the observations particularly interesting for long 
transported particle plumes. The data used to produce the statistics consist of 33 correlative observations that generate 13 monthly profiles. Figure 2a displays the mean extinction coefficient profiles for both systems: the discrepancy below 2 $\mathrm{km}$ is significantly high (mean extinction bias $0.05 \mathrm{~km}^{-1}$ ), on the other hand the differences at the upper levels are reduced (mean extinction bias less than $-0.01 \mathrm{~km}^{-1}$ ). The lower level disparity typically is weakened during summer months, and is exacerbated in winter, yet the sample size is small to quantify the periodicity of this discrepancy. Figure $2 b$ shows the comparison of CALIPSO against ground-based profiles for the backscatter coefficient at $532 \mathrm{~nm}$. The low level disparity, here, is significantly reduced. The mean relative extinction bias for the range $1-3 \mathrm{~km}$ is $40 \%$ while for backscatter is limited to $15 \%$. However, this CALIPSO underestimation is in agreement with what observed for Potenza already in CALIPSO Level 1 data [7].

CALIPSO aerosol retrieval relies on the lidar ratio assignment, which in turn is based on the aerosol type classification. Therefore the lidar ratio profile for each month is estimated and directly compared to averaged unconstrained EARLINET lidar ratio profile. Figure 2c reports mean profiles of the lidar ratio measured by EARLINET station and the mean profile of values assumed by the CALIPSO algorithm for the inversion. The CALIPSO lidar ratio is for the whole altitude range $S_{\text {aer }}=49.1 \pm 3.3$ sr. On the other hand EARLINET measured lidar ratio exhibits higher values in the range $1.5-2.7 \mathrm{~km}, \mathrm{~S}_{\mathrm{arr}}=61.5 \pm 3.4 \mathrm{sr}$, most likely because of the influence of more absorbing particles or the dust lidar ratio assignment that leads to CALIPSO lower values. In the height range $2.7-5 \mathrm{~km}$, the agreement is satisfactory with an EARLINET mean value of $\mathrm{S}_{\mathrm{aer}}=50.4 \pm 5.2 \mathrm{sr}$, suggesting that all the aerosol types identified compensate for this situation or the aerosol fields over the grid are frequently dust affected. The latter stems from EARLINET based studies, such as the study of [8] where the authors used a multiyear dust database and found values lidar ratio of $\sim 50 \mathrm{sr}$ for the site of Potenza.

Figure 3 reports the classification of aerosol features of the CALIPSO overpasses and the corresponding Potenza station observations. The investigated altitude range was partitioned into 1 $\mathrm{km}$ intervals and the percentage of layers identified within each bin is reported. Therefore, according to the boundary location, layers can be present in more than one height bin range.
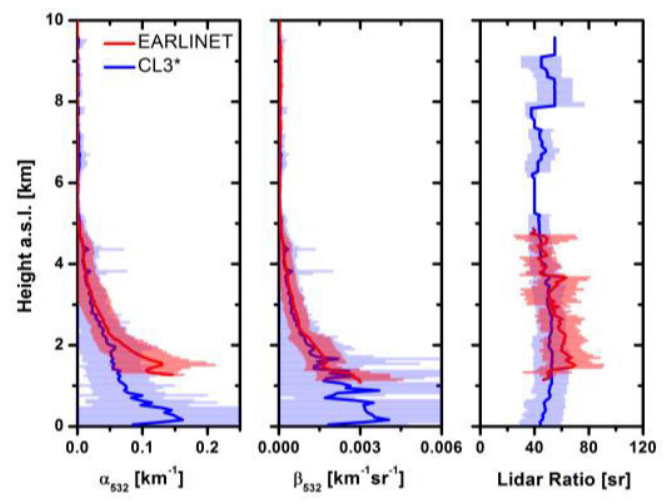

Figure 2: (a) extinction coefficient, (b) backscatter coefficient and (c) lidar ratio profiles at $532 \mathrm{~nm}$.

Figure $3 \mathrm{~b}$ gives an outlook of the aerosol types observed by CALIPSO. Dust and Polluted Dust prevail over the grid and moreover, present complementary behavior as Polluted Dust in the $1-2 \mathrm{~km}$ bin is the most important aerosol type and above that height pure Dust becomes the most important type. Smoke in the range 1-4 km keeps a constant presence. Some Polluted Continental cases are in the first height bin of the figure, and Clean Continental resides at the higher altitudes. As far as Clean Marine particles, they slightly affect the study area, yet below $3 \mathrm{~km}$ layers are identified as the ground track might be as well over the Mediterranean Sea (see Fig. 1).

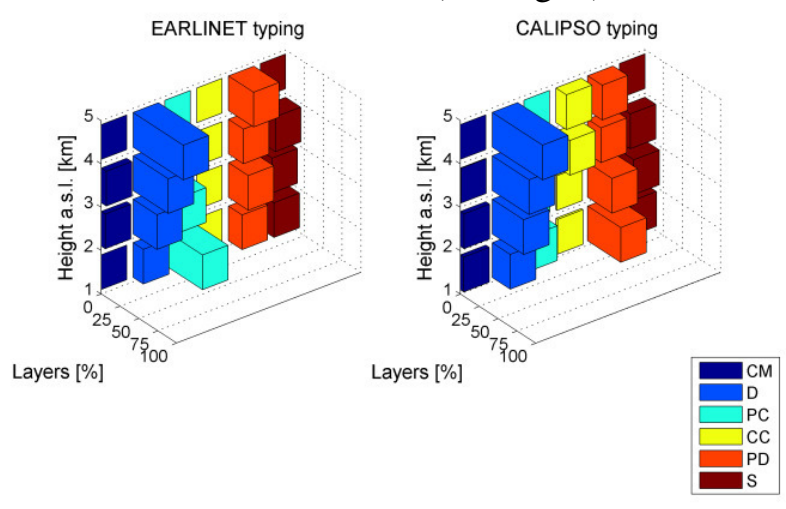

Figure 3: (a) EARLINET and (b) CALIPSO typing vertical proportioning for Potenza grid cell, the range interval is equal to $1 \mathrm{~km}$. CM stands for Clean Marine, D for Dust, PC for Polluted Continental, CC for Clean Continental, PD for Polluted Dust, and S for Smoke. 
Figure $3 \mathrm{a}$ presents the results of the typing procedure for the EARLINET correlative measurements (e.g., [9]; [10]). Again Dust and Polluted Dust affect the area around the site, although Polluted Continental particles in the first bin alter the aerosol situation. The latter can be ascribed to aerosols of anthropogenic origin within the nocturnal PBL and the adjoining residual layer around the area of Potenza site. Smoke particles, as was for the CALIPSO typing, have a range invariant character up to $4 \mathrm{~km}$. Polluted Dust by contrast to CALIPSO, produces a rather constant influence with height. Summarizing the Dust, Polluted Dust (apart from PBL) and Smoke components were effectively identified by CALIPSO.

\section{CONCLUSIONS}

The comparison based on CALIPSO correlative measurements is essential to understand if CALIPSO measurements are representative of the corresponding station surrounding area in a climatological sense and if there are systematic deviations due to assumptions in the CALIPSO retrievals.

Besides the Potenza station, 4 more EARLINET stations were examined. In particular a subtle underestimation mainly in the PBL and its adjoining regions was found, with a relative AOD difference of -0.016 . Regarding the backscatter comparison the relative difference in respect to the extinction comparison improved from $15.2 \%$ to $11.4 \%$. Hence, using the CALIPSO backscatter coefficient, the comparison improves the relative mean biases when compared to the CALIPSO extinction coefficient. The better agreement in terms of backscatter has to be ascribed to the higher impact of lidar ratio assumption on extinction rather than on backscatter. Moreover, the realized database gives the possibility to investigate the performance of the lidar ratio assignment CALIPSO scheme.

\section{ACKNOWLEDGEMENTS}

The financial support by the European Community through the ACTRIS Research Infrastructure Action under the $7^{\text {th }}$ Framework Programme under ACTRIS Grant Agreement $n \circ 262254$ is gratefully acknowledged.

\section{REFERENCES}

[1] Winker, D., et al., 2013: The global 3-d distribution of tropospheric aerosols as characterized by CALIOP. Atmos. Chem. Phys., 13, 3345-3361.

[2] Pappalardo, G., et al., 2010: EARLINET correlative measurements for CALIPSO: First intercomparison results. J. Geophys. Res., 115, D00H19.

[3] Wandinger, U., et al., 2011: Aerosols and Clouds: long-term Database from Spaceborne Lidar Measurements. Technical report, ESA Publications Division. Final report, ESTEC Contract 21487/08/NL/HE, Noordwijk, the Netherlands.

[4] Burton, S., et al., 2013: Aerosol classification from airborne HSRL and comparisons with the CALIPSO vertical feature mask, Atmos. Meas. Tech., 6(5),1397-1412.

[5] Amiridis, V., et al., 2013: Optimizing CALIPSO Saharan dust retrievals, Atmos. Chem. Phys., 13, 12089-12106.

[6] Campbell, J., et al, 2012: Evaluating nighttime CALIOP 0.532 um aerosol optical depth and extinction coefficient retrievals, Atmos. Meas. Tech., 5, 2143-2160.

[7] Mona, L., et al., 2009: One year of CNRIMAA multi-wavelength Raman lidar measurements in correspondence of CALIPSO overpass: Level 1 products comparison, Atmos. Chem. Phys., 9, 7213-7228.

[8] Mona, L., et al., 2014: EARLINET dust observations vs. BSC-DREAM8b modeled profiles: 12-year-long systematic comparison at Potenza, Italy. Atmos. Chem. Phys., 14, 87818793.

[9] Groß, S., et al., 2011: Characterization of Saharan dust, marine aerosols and mixtures of biomass burning aerosols and dust by means of multi-wavelength depolarization and Raman measurements during SAMUM-2, Tellus B, 63, 706-724.

[10] Mona, L., et al., 2012: Multi-wavelength Raman lidar observations of the Eyjafjallajökull volcanic cloud over Potenza, southern Italy, Atmos. Chem. Phys., 12, 2229-2244. 